\title{
ON MATRIX EXPONENTIAL APPROXIMATIONS OF RUIN PROBABILITIES FOR THE CLASSIC AND BROWNIAN PERTURBED CRAMÉR-LUNDBERG PROCESSES
}

\author{
F. AVRAM AND M. PISTORIUS
}

\begin{abstract}
Padé rational approximations are a very convenient approximation tool, due to the easiness of obtaining them, as solutions of linear systems. Not surprisingly, many matrix exponential approximations used in applied probability are particular cases of first and second order "admissible Padé approximations" of a Laplace transform, where admissible stands for nonnegative in the case of a density, and for nonincreasing in the case of a ccdf (survival function).

Our first contribution below is the observation that for CramérLundberg processes and Brownian perturbed Cramér-Lundberg processes there are three distinct rationally approximations of the Pollaczek-Khinchine transform, corresponding to approximating a) the claims transform, b) the stationary excess transform, and c) the aggregate loss transform.

A second contribution is providing three new always admissible second order approximations for the ruin probabilities of the Cramér-Lundberg process with Brownian perturbation, one of which reduces in the absence of perturbation to DeVylder's approximation.

Our third contribution is a method for comparing the resulting approximations, based on the concept of largest weak-admissibility interval of the compounding/traffic intensity parameter $\rho$.
\end{abstract}

Keywords: Pollaczek-Khinchine formula; incomplete information; matrix exponential distributions; Padé approximation; perturbed CramérLundberg process; DeVylder's approximation

\section{Introduction: The PollaczeK-Khinchine formula}

We provide below approximations for the law of the supremum of a Brownian perturbed Cramér-Lundberg risk processes Schmidli (1999) defined by

$$
X(t)=u+c t-\sum_{i=1}^{N_{\lambda}(t)} Z_{i}+\sigma B(t),
$$


where $Z_{i}, i \geq 1$ are i.i.d. random variables representing the claims, $N_{\lambda}(t)$ is an independent Poisson process with exponential inter-arrival times of rate $\lambda$, and $\sigma B(t), \sigma>0$ is an independent Brownian perturbation of mean 0 and variance $\sigma^{2}$.

The power series expansion of the Laplace exponent/cumulant generating function/symbol $\kappa(s)=\log \left(\mathbb{E} \mathrm{e}^{s(X(1)-X(0))}\right)$ is:

$(2) \kappa(s)=p s+\frac{\eta_{2, \sigma}}{2} s^{2}+\sum_{k=3}^{\infty}(-s)^{k} \frac{\lambda m_{k}}{k !}=\frac{\sigma^{2} s}{2}(\tilde{c}-\tilde{\lambda} \widehat{\bar{F}}(s)+s)$,

where $m_{k}=\mathbb{E}\left[Z_{i}^{k}\right]$ are the claim moments, where

$$
p:=c-\lambda m_{1}=\lambda m_{1} \theta>0
$$

is the Levy drift/profit rate, $\eta_{2, \sigma}=\lambda m_{2}+\sigma^{2}, \tilde{c}=c / \frac{\sigma^{2}}{2}, \tilde{\lambda}=\lambda / \frac{\sigma^{2}}{2}$, and $\widehat{\bar{F}}(s)$ denotes the Laplace transform of the survival function of the claims.

Three objects of central interest in ruin theory are:

- The distribution of the maximal aggregate loss

$$
L=\max _{0 \leq t<\infty} \sum_{i=1}^{N_{\lambda}(t)} Z_{i}-c t-\sigma B(t)
$$

(the negative of the all-time infimum of the process $X$ started from 0$)$.

- The first passage time below 0:

$$
T(u):=\inf \{t \geq 0: X(t)<0\} .
$$

- The eventual ruin probability/survival function of $L$ :

$$
\Psi(u)=P[T(u)<\infty]=P[L>u] .
$$

The eventual ruin probability $\Psi(u)$ is not identically one iff the Levy $\mathrm{drift} /$ profit rate $(3)$ is positive, which will be assumed from now on.

The Pollaczek-Khinchine formula. The maximal aggregate loss may also be represented as a geometric compound sum (of "ladders"), and its Laplace transform is known as the Pollaczek-Khinchine formula see for example (Kyprianou, 2006). In the case of Brownian perturbed processes, putting $\tilde{\kappa}_{p}(s)=\frac{\sigma s}{2}$, this reads as:

$$
\begin{aligned}
\hat{\Psi}(s) & =\frac{1}{s}-\frac{\kappa^{\prime}(0)}{\kappa(s)} \\
& =\frac{\lambda\left(m_{1}-\hat{\bar{F}}(s)\right)+\tilde{\kappa}_{p}(s)}{s\left(c-\lambda \hat{\bar{F}}(s)+\tilde{\kappa}_{p}(s)\right)}=\frac{\rho\left(1-\hat{f}_{e}(s)\right)+\tilde{\kappa}_{p}(s)}{s\left(1-\rho \hat{f}_{e}(s)+\tilde{\kappa}_{p}(s)\right)},
\end{aligned}
$$


where $f_{e}(x)$ and $\hat{f}_{e}(s)$ denote the stationary excess density of the claims and its Laplace transform.

Remark 1. The Renyi, DeVylder, Whitt, Ramsay, and Badescu $\&$ Stanford approximations used in queueing and risk theory are all Padé approximations of the Pollaczek-Khinchine transform (6).

Remark 2. Our paper is motivated by the observation that rational approximation of this formula may be implemented either for the input transform $\hat{f}_{Z}(s)$, or for the stationary excess transform $\hat{f}_{e}(s)$, or for the final transform $\hat{\Psi}(s) \S$.

This is straightforward with Brownian perturbations, and could be achieved as well with other perturbations, once some rational approximation method for $\tilde{k}_{P}(s)$ is selected.

Contents. Our paper starts with a review of matrix exponential approximations in Section 2.

The classification in three types of Padé approximations of the PollaczekKhinchine transform, for: a) the claims transform, b) the stationary excess transform, and c) the aggregate loss transform is developped in Section 3 - see Theorem 1, and illustrated by the classic approximations of Renyi, DeVylder and Ramsay.

A review of the Cramér-Lundberg process with Brownian perturbation is provided in Section 4, and three new always admissible second order approximations for its ruin probabilities are given in Theorem 2, Section 5. One of these reduces when $\sigma=0$ to DeVylder's approximation for the classic Cramér-Lundberg process - see Remark 9. Note that higher order approximations are not at all easy to get (by any of the three methods), due to the difficulty of ensuring admissibility. These difficulty is illustrated by the sometimes admissible second order approximations for the non-perturbed Cramér-Lundberg process provided in Theorem 3.

One possible method for comparing the three approaches is to use the observation that the final approximation for ruin probabilities can be admissible (non-increasing) even when the claims density is not nonnegative. This suggests the concept of weak-admissibility set $I$ of all values of the compounding/traffic intensity parameter $\rho$ which render the final approximation admissible - see Section 6- and allows

\footnotetext{
§Following Abate and Whitt (1996), we may say that the Pollaczek-Khinchine formula is a composition of the "stationary excess operator" $f_{Z}->f_{e}$, and of the "geometric compounding operator", applied successively to the input density $f_{Z}(x)$, and one may use rational approximation at any step of this chain.
} 
us to compare the three approximations by the size of their weakadmissibility set. We undertake this comparison for the classic CramérLundberg process with uniformly distributed claims in Section 6.

\section{MATRIX EXPONENTIAL APPROXIMATIONS}

Approximation by exponential polynomials. The calculation of many quantities of interest in applied probability (ruin theory, queueing, reliability, mathematical finance, etc.) is more convenient when the input data have an "exponential-polynomial" density of the form

$$
f(t)= \begin{cases}\sum_{k=0}^{K} w_{k} e^{-\xi_{k} t} \geq 0, & \forall t \geq 0 \\ 0, & \forall t<0\end{cases}
$$

with $\xi_{k}$ having non-negative real part, and $\omega_{k}$ being polynomials.

When $\omega_{k}$ are constants, the densities (7) are called generalized hyperexponential (GHE), the word generalized being a reference to the fact that no assumption is made on the sign of $\omega_{k}$, except for the global nonnegativity of $f(t)$. Allowing polynomials $\omega_{k}(t)$ rather than constants is however necessary in order to have a closed approximation space, since the possible "collision" of exponents requires including the derivatives $\frac{\partial e^{-\xi t}}{\partial \xi}=t e^{-\xi t}, \ldots$

If $f(t)$ is observed on an equally spaced sample, (7) may be viewed as a hypothesis on the fact that the rank of increasing Hankel matrices based on the data stabilizes at the value $K+1$, cf. a theorem due to Kronecker. The determination of the parameters $\omega_{k}, \xi_{k}$ disregarding the non-negativity constraint is the famous De Prony problem (Prony, 1795; Potts and Tasche, 2012; Andersson et al., 2011).

Admissible rational Laplace transform approximations. Equivalently, (7) amounts to approximating the empirical Laplace transform by a rational function

$$
I^{-1} \sum_{i=0}^{I} e^{-s X_{i}} \approx \frac{\sum_{i=0}^{n-1} a_{i} s^{i}}{\sum_{i=0}^{n} b_{i} s^{i}} .
$$

Hence, in the Laplace domain, the problem is: find rational approximations of a given Laplace transform, with admissible (nonnegative) inverse.

The approximations (7)-(8) are known under various names: Dirichlet polynomials (Martin and Shubov, 1993), exponential polynomials, rational Laplace transform approximations (RLTA), and exponential sums. In probability the name in use is "matrix exponential functions", stressing the importance of the matrix parametrization (9). 
The matrix-exponential parametrization. Note that exponential polynomials are uniquely characterized by a minimal scalar ODE with constant coefficients, and by initial conditions. Converting the scalar ODE to a linear ODE system with constant coefficients yields the parametrization (9).

Definition 1. PH (phase-type) and ME (matrix-exponential) densities: a) A continuous density on $[0, \infty)$ is called of phase-type $P H(\boldsymbol{\alpha}, A)$ if

$$
f(t)=\boldsymbol{\alpha} e^{A t} \boldsymbol{a}, \forall t \geq 0 \Leftrightarrow \hat{f}(s)=\boldsymbol{\alpha}(s I-A)^{-1} \boldsymbol{a},
$$

with

(1) $\boldsymbol{\alpha}$ a probability row vector,

(2) A a subgenerator matrix, satisfying $A_{i j} \geq 0$ for $i \neq j, A \mathbf{1} \leq \mathbf{0}$, and

(3) $\boldsymbol{a}=-A$ 1, 1 are column vectors.

These assumptions imply that $f(t)$ is a density and so $f(t) \geq 0, \forall t \geq$ 0 .

b) When a representation (9) exists, but the components $\boldsymbol{\alpha}, A, \boldsymbol{a}$ are not necessarily as in part a) (and thus $f(t) \geq 0, \forall t \geq 0$ may not hold), we will call $f(t)$ a matrix exponential function.

c) A matrix exponential density is a matrix exponential function $f(t)$ defined by (9), but without any assumptions on the components $\boldsymbol{\alpha}, A, \boldsymbol{a}$, except that

$$
f(t) \geq 0, \forall t \geq 0 .
$$

Moreover, the total mass is assumed to be less than one.

Equivalently, the "survival/complementary cumulative function" $\bar{F}(t)$ : $\int_{t}^{\infty} f(x) d x$ must have a representation

$$
\bar{F}(t)=\boldsymbol{\alpha} e^{t A} \mathbf{1}, \quad t>0, \quad \bar{F}(t) \text { nonincreasing. }
$$

We will refer to both the case $f(t)$ non-negative and $\bar{F}(t)$ nonincreasing as "admissible".

d) We say that a random variable $X$ has a matrix exponential distribution iff it has a rational Laplace transform with numerator and denominator of equal degree

$$
E\left[e^{-s X}\right]=a_{n}+\left(1-a_{n}\right) \frac{\sum_{i=0}^{n-1} a_{i} s^{i}}{\sum_{i=0}^{n} b_{i} s^{i}}
$$

(this representation puts in evidence that $X$ must have an atom at 0 of mass $a_{n}$, and a continuous part with matrix exponential density of mass $1-a_{n}$ ). 
The set of matrix exponential distributions with degree of the denominator in a simplified form of the Laplace transform $n$ (McMillan degree) will be denoted by $\mathcal{M} E_{n}$.

Matrix exponential densities on $\mathbb{R}_{+}(9)$ (or, equivalently, densities with rational Laplace transform) have been at the center of research in applied probability and engineering for a long while, starting maybe with Erlang (Erlang, 1909; Neuts, 1981).

This is due to the fact that applied probability deals often with "Markovian black boxes", having as input the empirical measure of observed data, and having complicated outputs. For example, the output may consist of the density of a performance measure of interest, obtainable by solving integro-differential equations. The assumption that the theoretical density of the observed data is a combination of exponentials (which are eigenfunctions of the derivative operator D) may simplify the problem and render unnecessary the resolution of the integro-differential equations.

The best known example is that of computing the survival function of the maximum of a compound Poisson sum with a linear drift

$$
\Psi(u)=P\left[\max _{t \geq 0}\left(\sum_{k=1}^{N_{\lambda}(t)} Z_{k}-c t\right)>u\right]
$$

Note that (12) provides the ruin probability of a Cramér-Lundberg process, and also the probability that the stationary waiting time in the $\mathrm{M} / \mathrm{G} / \mathrm{l}$ queue with the same arrival process and service times distributed as the claim sizes is larger than $u$ (Asmussen and Rolski, 1992, (1.2)) (with $c=1$ ).

When the density of the claims is an exponential polynomial, the same will be true for $\Psi(u)$ :

$$
\Psi(u)=\sum_{k} C_{k} e^{-\gamma_{k} u}
$$

where $\gamma_{k}$ are the roots of the Cramér Lundberg equation $k(-s)=0$, and $C_{k}$ are the coefficients of the partial fractions decomposition of (6). Note that when the roots $\gamma_{k}$ are not distinct, the coefficients $C_{k}$ become polynomials.

The explicit formula (13) may be more parsimoniously described and implemented using matrix exponential parametrizations (9) of the input and output densities. Indeed, when the claims $Z_{i}$ have a phasetype distribution of type $(\boldsymbol{\beta}, B)$, it holds that:

$$
\Psi(u)=\boldsymbol{\rho} e^{u(B+\boldsymbol{b} \boldsymbol{\rho})} \mathbf{1}
$$


where $\boldsymbol{\rho}=\frac{\lambda}{c} \boldsymbol{\beta}(-B)^{-1}$ is a row vector, and $\mathbf{1}=(1,1, \ldots, 1)^{t}, \boldsymbol{b}=(-B) \mathbf{1}$ are column vectors (Asmussen and Rolski, 1992, (3.1)), (Avram and Usabel, 2003, (10)).

Remark 3. Note that powerful generalizations of this formula are available for obtaining the density of the ruin time for the SparreAndersen model with PH claims (with inter-arrivals being an arbitrary i.i.d. sequence) - see for example (?Frostig et al., 2012). Therefore, quite difficult problems may be solved efficiently by providing a matrix exponential approximation of the density of the data.

Remark 4. Numerically, the matrix exponential parametrization is in line with replacing root finding by computing the eigenvalues of the companion matrix. This is a "symbolic-numeric" (i.e. an essentially errorfree) operation. Note also that matrix exponentiation may be achieved symbolically, via the Cayley-Hamilton theorem or Putzer's method.

Padé/moments approximations. Another attraction of matrix exponential densities is that of providing simple density approximations in the case of few data, when only some moments of the input distribution may be safely assumed to be known ${ }^{\S}$. This is in line with the venerable and easy implementable Padé approximation.

A yet another attraction is that of cases like that of retrial queues, where the integro-differential equation is complicated enough to force restricting to a few moments - see (Nobel and Tijms, 2006; Kim and Kim, 2011, 2013).

Admissibility of Padé approximations. The admissibility of Padé approximations is already non-trivial at second order. Let

$$
\hat{f}(s)=\frac{a_{0}+a_{1} s}{b_{0}+b_{1} s+b_{2} s^{2}}=\frac{a_{0}(1+a s)}{b_{0}+b_{1} s+b_{2} s^{2}}
$$

denote the second order Padé $(1,2)$ approximation of a transform $\hat{f}(s)$. When $f(x)$ is a density, we must have $a_{0}=b_{0}$. Furthermore, as well known and easy to check, $a_{1}=b_{1}-b_{0} \mu_{1}$, and the quadratic polynomial $b(s)$ in the denominator may be written in terms of the first three moments as

$$
b(s)=b_{0}+b_{1} s+b_{2} s^{2}=\left(\begin{array}{ccc}
1 & \mu_{1} & \mu_{2} \\
\mu_{1} & \mu_{2} & \mu_{3} \\
s^{2} & -s & 1
\end{array}\right)
$$

\footnotetext{
${ }^{\S}$ with more data, using the empirical density as input is much more efficient than using a few moments
} 
with "Hankel" coefficients:

$$
b_{2}=H_{2,3}=\mu_{1} \mu_{3}-\mu_{2}^{2}, b_{1}=H_{1,3}=\mu_{3}-\mu_{1} \mu_{2}, b_{0}=H_{1,2}=\mu_{2}-\mu_{1}^{2}
$$

When $b_{0}>0$ (which holds in our case for nondeterministic claims), such an approximation is admissible iff

(1) $b_{2}>0$, the discriminant of the denominator is nonnegative and both roots are negative, which requires $b_{1} \geq 2 \sqrt{b_{2} b_{0}}$,

(2) $a:=\frac{a_{1}}{a_{0}}=\frac{b_{1}}{b_{0}}-\mu_{1}>0$,

(3) at least one of the roots of $b(s)$ is bigger than $-a$, which requires $b(-a) \leq 0$ or $\left(b(-a)>0\right.$ and $\left.b^{\prime}(-a) \leq 0\right)$.

The domain where these conditions hold is $\mathcal{D}=\left\{\bar{b}_{2}>0, \bar{b}_{1}>0\right\} \cap$ $\left(\mathcal{D}_{1} \cup \mathcal{D}_{2}\right)$, where

$$
\mathcal{D}_{1}=\left\{\begin{array}{l}
\frac{2 b_{0}}{b_{1}}+\mu_{1} \leq \frac{b_{1}}{b_{0}} \\
4 b_{2} \leq \frac{b_{1}^{2}}{b_{0}}
\end{array} \quad \mathcal{D}_{2}=\left\{\begin{array}{l}
\frac{b_{0}}{b_{1}}+\mu_{1}<\frac{b_{1}}{b_{0}} \leq \frac{2 b_{0}}{b_{1}}+\mu_{1} \\
b_{2} \leq \frac{b_{0}\left(b_{1}^{2}-b_{0}^{2}-b_{1} \mu_{1} b_{0}\right)}{\left(b_{1}-b_{0} \mu_{1}\right)^{2}}
\end{array}\right.\right.
$$

In terms of $\bar{b}_{i}=\frac{b_{i}}{b_{0}}, i=1,2$ and $a=\frac{a_{i}}{a_{0}}>0$, the domain is:

$$
\mathcal{D}_{1}=\left\{\begin{array}{l}
2 \leq a \bar{b}_{1} \\
4 \bar{b}_{2} \leq \bar{b}_{1}^{2}
\end{array} \quad \mathcal{D}_{2}=\left\{\begin{array}{l}
1 \leq a \bar{b}_{1} \leq 2 \\
a^{2} \bar{b}_{2}+1 \leq a \bar{b}_{1}
\end{array}\right.\right.
$$

In terms of moments, one may apply (Telek and Heindl, 2002; Bobbio et al., 2005)

$$
\begin{cases}\left(3 \bar{\mu}_{2}-2\right)+2 \sqrt{\left(1-\bar{\mu}_{2}\right)^{3}}<\bar{\mu}_{3} \leq c_{v}=2 \bar{\mu}_{2}-1 & \frac{3}{4}<\bar{\mu}_{2}<1 \\ \bar{\mu}_{3}>\bar{\mu}_{2}^{2} & \bar{\mu}_{2}>1\end{cases}
$$

where $\bar{\mu}_{i}=\frac{\mu_{i}}{\mu_{1}^{i}}$.

Admissibility of high order Padé approximations. The "modified Hankel" structure in (15) is in fact valid for any order. Higher order Padé (and Chebyshev-Padé) approximations are thus quite easy to get, but ensuring the non-negativity or monotonicity of their inverses leads to complicated conditions. For order $n \geq 4$, the geometry of the corresponding admissibility domain is yet to be determined.

The first practical approximate tests for the non-negativity of high order given exponential polynomial densities became available only recently, with the command CheckMEPositiveDensity of the package BUTools (Reinecke and Telek, 2013), and the package SOPE (Dumitrescu et al., 2014).

Remark 5. The optimization package SOPE (Dumitrescu et al., 2014) www.schur.pub.ro/sope allows replacing a non-admissible exponential 
polynomial with real exponents by the closest $L_{2}$ admissible exponential polynomial with the same exponents.

\section{Three Padé approximations for the RUin probabilities OF THE Classic CRAMÉR-LundBERG PROCESS}

In this section, we make the apparently new observation that there are three ways to construct Padé approximations for the Laplace transform $\hat{\Psi}(s)$, depending on whether one imposes or not the known initial conditions for $\Psi(0)$ and $\Psi^{\prime}(0)$. The three resulting strategies are:

(1) classic Padé, using the first $2 n$ ruin moments $\Psi_{0}=\frac{\tilde{m}_{1}}{\theta}, \Psi_{1}=$ $\frac{\tilde{m}_{2}}{2 \theta}+\left(\frac{\tilde{m}_{1}}{\theta}\right)^{2}, \ldots, \Psi_{2 n-1}$. Alternatively, one may set up a system for the Padé $[n-1, n]$ approximation.

For example, at $n=1$, we find:

$$
\begin{aligned}
& \hat{\Psi}(s)=\frac{1}{s}-\frac{p}{s\left(p+\lambda m_{2} s / 2-\lambda m_{3} s^{2} / 6+\ldots\right)}= \\
& \frac{\lambda m_{2} / 2-\lambda m_{3} s / 6+\ldots}{p+\lambda m_{2} s / 2-\lambda m_{3} s^{2} / 6+\ldots} \approx \frac{a}{s+b} \\
& \Leftrightarrow a s\left(p+\lambda m_{2} s / 2-\lambda m_{3} s^{2} / 6+\ldots\right) \\
& \approx(s+b)\left(\lambda m_{2} s / 2-\lambda m_{3} s^{2} / 6+\ldots\right) \\
& \Leftrightarrow a p=b \lambda m_{2} / 2, a m_{2} / 2=m_{2} / 2-b m_{3} / 6 \\
& \Leftrightarrow a=\frac{3 \lambda m_{2}^{2}}{3 \lambda m_{2}^{2}+2 p m_{3}}, b=\frac{6 p m_{2}}{3 \lambda m_{2}^{2}+2 p m_{3}}
\end{aligned}
$$

This yields the famous De Vylder approximation

$$
\Psi(x) \approx a e^{-b x}
$$

It works well for large $x$ (Grandell, 2000), but doesn't fit the known values of $\Psi(0), \Psi^{\prime}(0)$.

Since we are approximating the survival function of $L$ which is representable as a geometric compound sum, we will call this approach the geometric compound approximation.

(2) two point Padé, using the first $2 n-1$ moments $\Psi_{k}, k=0,1, \ldots, 2 n-$ 2 plus the condition $\Psi(0)=\rho$. This is equivalent to using the stationary excess moments $\tilde{m}_{k}, k=0,1, \ldots, 2 n-1$ of $\hat{f}_{e}(s)-$ see Theorem 1 below- and will be called stationary excess approximation.

(3) two point Padé, using the first $2 n-2$ moments $\Psi_{k}=\int_{0}^{\infty} x^{k} \Psi(x) d x, k=$ $0,1, \ldots, 2 n-3$ plus the two conditions $\Psi(0)=\rho, \Psi^{\prime}(0)=-\frac{\rho(1-\rho)}{m_{1}}$. This is equivalent to fitting the claims moments $m_{k}, k=0,1, \ldots, 2 n-$ 
1 - see Theorem 1 below, and will be called input approximation.

\section{Theorem 1. Three Padé approximations of ruin probabilities for the Cramér-Lundberg process.}

a) The stationary excess approximation. Fitting the first $2 n-1$ moments $\Psi_{k}=\int_{0}^{\infty} x^{k} \Psi(x) d x, k=0,1, \ldots, 2 n-2$ plus the condition $\Psi(0)=\rho$ is equivalent to fitting $\hat{f}_{e}(s)$ from the first $2 n$ moments $\tilde{m}_{k}=$ $\frac{m_{k+1}}{(k+1) m_{1}}, k=0,1, \ldots, 2 n-1$ of $f_{e}(x)$, and then inserting $\hat{f}_{e}(s)$ in the Pollaczek-Khinchine formula.

b) The input density approximation. The Padé $(n-1, n)$ approximation obtained by fitting the first $2 n$ moments $m_{k}, k=0,1, \ldots, 2 n-1$ of $f(x)$, and inserting $\hat{f}(s)$ in the Pollaczek-Khinchine formula, is equivalent to either of:

(1) fitting $\hat{f}_{e}(s)$ from the first $2 n-1$ moments $\tilde{m}_{k}=\frac{m_{k+1}}{(k+1) m_{1}}, k=$ $0,1, \ldots, 2 n-2$ of $f_{e}(x)$ and the condition $f_{e}(0)=\frac{1}{m_{1}}$, and then inserting $\hat{f}_{e}(s)$ in the Pollaczek-Khinchine formula.

(2) fitting the first $2 n-2$ moments $\Psi_{k}, k=0, \ldots, 2 n-3$ of $\Psi(x)$, plus the initial conditions

$$
\begin{aligned}
& \Psi(0)=\lim _{s \rightarrow \infty} s \hat{\Psi}(s)=\rho \\
& \Psi^{\prime}(0)=-\frac{\rho(1-\rho)}{m_{1}} \Leftrightarrow f_{e}(0)=\frac{1}{m_{1}} .
\end{aligned}
$$

Proof: We sketch now the proof of (b)(2) (the proofs of the other two points are similar and omitted). Let $a(s) / b(s)$ denote an approximation of the input density transform, and check that the corresponding Padé approximation of the stationary excess density transform $\hat{f}_{e}(s)=\frac{1-\hat{f}(s)}{\mu_{1} s}$ may be written as indicated in Box 1, and satisfies the limiting relation there.

$$
\begin{aligned}
& \hat{f}_{e}(s)=\frac{b(s)-a(s)}{\mu_{1} s b(s)} \\
& =\frac{b_{0}+s b_{1}+\ldots+b_{n} s^{n}-\left(b_{0}+\left(b_{1}-b_{0} \mu_{1}\right) s+\ldots\left(b_{n-1}-b_{n-2} \mu_{1}+\ldots(-1)^{n-1} \mu_{n-1} b_{0}\right) s^{n-1}\right)}{\mu_{1} s b(s)} \\
& =\frac{b_{0}+\left(b_{1}-b_{0} \mu_{2} / \mu_{1}\right) s+\ldots+b_{n} / \mu_{1} s^{n-1}}{b(s)} \Longrightarrow f_{e}(0)=\lim _{s \rightarrow \infty} s \hat{f}_{e}(s)=\frac{1}{\mu_{1}}
\end{aligned}
$$


The result follows then from:

$$
\begin{aligned}
& s \hat{\Psi}(s)=\rho \frac{1-\hat{f}_{e}(s)}{1-\rho \hat{f}_{e}(s)} \underset{s \rightarrow \infty}{\Longrightarrow} \rho \\
& s(s \hat{\Psi}(s)-\rho)=-\rho(1-\rho) \frac{s \hat{f}_{e}(s)}{1-\rho \hat{f}_{e}(s)} \underset{s \rightarrow \infty}{\Longrightarrow}-\frac{\rho(1-\rho)}{m_{1}}
\end{aligned}
$$

Remark 6. All the three approaches above may be viewed as classic Padé approximations, applied respectively to $\hat{\Psi}(s)$, the equilibrium transform $\hat{f}_{e}(s)$ or the claims $\hat{f}(s)$ transform.

All the classic exponential mixture approximations recalled in the abstract turn out to be particular cases of our three schemes.

Remark 7. It is possible to use any rational approximation of the Laplace transform (like (Johnson and Taaffe, 1989), admissible three moments approximations (Bobbio et al., 2005), etc), at all the three levels.

Example 1. (1) For $n=1, \hat{f}(s)=\hat{f}_{e}(s) \approx \frac{1}{1+s m_{1}}$. The input density Padé $(0,1)$ approximation of $\hat{f}(s)$ is:

$$
\begin{aligned}
& \hat{\Psi}(s) \approx \frac{\rho}{s} \frac{1-\frac{1}{1+s m_{1}}}{1-\rho \frac{1}{1+s m_{1}}}=\frac{\rho}{s+(1-\rho) / m_{1}} \Leftrightarrow \\
& \Psi(x) \approx \rho \mathrm{e}^{-x(1-\rho) / m_{1}} .
\end{aligned}
$$

It fits $\Psi(0), \Psi^{\prime}(0)$.

(2) The $(0,1)$ stationary excess approximation based on $\hat{f}_{e}(s) \approx$ $\frac{1}{1+s \tilde{m}_{1}}$ yields:

$$
\hat{\Psi}(s) \approx \frac{\rho}{s+(1-\rho) / \tilde{m}_{1}} \Leftrightarrow \Psi(x) \approx \rho \mathrm{e}^{-x(1-\rho) / \tilde{m}_{1}} .
$$

This is also known as the Renyi approximation, and it is exact asymptotically in the limit $\rho \rightarrow 1$ when $\tilde{m}_{1}$ exists see (Kalashnikov, 1997), (Grandell, 2000, (31)). It fits $\Psi(0)$, but it doesn't fit $\Psi^{\prime}(0)$.

\section{The Brownian Perturbed Cramér-Lundberg process}

For a perturbed process we have, besides $\Psi(u)$, two unknowns of interest: the probability of "creeping ruin" by diffusion $\Psi_{c}(u)$, and that of "ruin by jump" $\Psi_{j}(u)$. The respective IDE's and "obvious boundary 
conditions" are:

$$
\begin{array}{cc}
G \Psi_{j}(u)+\lambda \bar{F}(u)=0 & \Psi_{j}(0)=0 \\
G \Psi_{c}(u)=0, & \Psi_{c}(0)=1 \\
G \Psi(u)+\lambda \bar{F}(u)=0, & \Psi(0)=1
\end{array}
$$

where $G$ is the infinitesimal generator of our process - see for example (Feng, 2011).

Taking Laplace transform, putting $D=\frac{\sigma^{2}}{2}$ and using the fact that the factor $s$ of $\kappa(s)$ must appear also in the RHS (since the positive loading condition implies that $s=0$ is not a singularity) yields

$$
\begin{array}{cl}
\kappa(s) \hat{\Psi}_{j}(s)=D\left(\Psi_{j}^{\prime}(0)\right)-\lambda \hat{\bar{F}}(s), & D \Psi_{j}^{\prime}(0)-\lambda m_{1}=0 \\
\kappa(s) \hat{\Psi}_{c}(s)=D\left(s+\Psi_{c}^{\prime}(0)\right)+c, & D \Psi_{c}^{\prime}(0)+c=0 \\
\kappa(s) \hat{\Psi}(s)=D\left(s+\Psi^{\prime}(0)\right)+c-\lambda \hat{\bar{F}}(s), & D \Psi^{\prime}(0)+c-\lambda m_{1}=0
\end{array}
$$

Solving for the Laplace transforms yields:

$$
\begin{aligned}
& \hat{\Psi}_{j}(s)=\frac{\tilde{\lambda}\left(m_{1}-\hat{\bar{F}}(s)\right)}{\kappa(s)}=\frac{\lambda\left(m_{1}-\hat{\bar{F}}(s)\right)}{s(s+\tilde{c}-\tilde{\lambda} \hat{\bar{F}}(s))} \\
& \hat{\Psi}_{c}(s)=\frac{D s}{\kappa(s)}=\frac{D}{D s+c-\lambda \hat{\bar{F}}(s)}=\frac{1}{s+\tilde{c}-\tilde{\lambda} \hat{\bar{F}}(s)} \\
& \hat{\Psi}(s)=\frac{D s-\lambda\left(\hat{\bar{F}}(s)-m_{1}\right)}{\kappa(s)} \Leftrightarrow \hat{\bar{\Psi}}(s)=\frac{c-\lambda m_{1}}{\kappa(s)}=\frac{\kappa^{\prime}(0)}{\kappa(s)},
\end{aligned}
$$

where $\tilde{\lambda}, \tilde{c}$ indicate scaled values divided by $D=\frac{\sigma^{2}}{2}$.

Remark 8. The last formula shows, as well-known, that the PollaczekKhinchine formula is insensitive to the form of the spectrally negative Levy process involved, when written in terms of the symbol $\kappa(s)$.

In conclusion, we recover the results of (Dufresne and Gerber, 1991).

Proposition 1. The probabilities of ruin by diffusion $\Psi_{c}(u)$ and by jump $\Psi_{j}(u)$ satisfy the boundary conditions

$$
\Psi_{c}(0)=1, \Psi_{j}(0)=0, \Psi_{c}^{\prime}(0)=-\tilde{c}, \Psi_{j}^{\prime}(0)=\tilde{\lambda} m_{1},
$$

and their Laplace transforms satisfy

$$
\begin{aligned}
& \tilde{p} \hat{\Psi}_{c}(s)=\phi(s), \\
& \hat{\Psi}_{j}(s)=\frac{1-\tilde{p} \hat{\Psi}_{c}(s)}{s}-\hat{\Psi}_{c}(s)=\frac{1-\phi(s)}{s}-\frac{\phi(s)}{\tilde{p}}
\end{aligned}
$$

where $\phi(s)=\frac{1-\rho}{1-\rho \hat{f}_{e}(s)+\frac{\sigma^{2}}{2 c} s}, \tilde{p}=\frac{p}{\sigma^{2} / 2}$. 
Example 2. For the perturbed Cramér-Lundberg process with exponential jumps of rate $\mu$, and Laplace exponent

$$
\kappa(s)=\frac{\sigma^{2} s^{2}}{2}+c s-\lambda \frac{s}{s+\mu},
$$

we find:

$$
\begin{aligned}
& \hat{\Psi}(s)=\frac{1}{s}-\frac{\kappa^{\prime}(0)}{\kappa(s)}=\frac{1}{s}-\frac{c-\lambda / \mu}{\frac{\sigma^{2}}{2} s^{2}+c s-\lambda \frac{s}{\mu+s}}=\frac{1}{s}\left(1-\frac{c-\lambda / \mu}{\frac{\sigma^{2}}{2} s+c-\frac{\lambda}{\mu+s}}\right) \\
& =\frac{\frac{\sigma^{2}}{2} s+\frac{\lambda s}{\mu(\mu+s)}}{s\left(\frac{\sigma^{2}}{2} s+c-\frac{\lambda}{\mu+s}\right)}=\frac{\frac{\sigma^{2}}{2}+\frac{\lambda}{\mu(\mu+s)}}{\frac{\sigma^{2}}{2} s+c-\frac{\lambda}{\mu+s}}=\frac{1+\frac{\tilde{\lambda}}{\mu(\mu+s)}}{s+\tilde{c}-\frac{\tilde{\tilde{H}}}{\mu+s}}=\frac{\mu+s+\frac{\tilde{\lambda}}{\mu}}{(\mu+s)(s+\tilde{c})-\tilde{\lambda}}, \\
& \hat{\Psi}_{c}(s)=\frac{\mu+s}{(s+\tilde{c})(\mu+s)-\tilde{\lambda}}=\frac{s+\mu}{\left(s+\gamma_{1}\right)\left(s+\gamma_{2}\right)}, \\
& \hat{\Psi}_{j}(s)=\hat{\Psi}(s)-\hat{\Psi}_{c}(s)=\frac{\frac{\tilde{\lambda}}{\mu}}{(\mu+s)(s+\tilde{c})-\tilde{\lambda}}=\frac{\frac{2 \lambda}{\sigma^{2} \mu}}{\left(s+\gamma_{1}\right)\left(s+\gamma_{2}\right)},
\end{aligned}
$$

where $\gamma_{1}, \gamma_{2}$ are the absolute values of the negative roots of the Cramér Lundberg equation:

$$
s^{2}+s(\tilde{c}+\mu)+\tilde{c} \mu-\tilde{\lambda}=0
$$

ordered so that $\gamma_{1}<\mu<\gamma_{2}$.

It follows that

$$
\begin{aligned}
\Psi_{j}(u) & =\frac{\frac{\tilde{\lambda}}{\mu}}{\gamma_{2}-\gamma_{1}}\left(e^{-\gamma_{1} u}-e^{-\gamma_{2} u}\right) \\
\Psi_{c}(u) & =\frac{\mu-\gamma_{1}}{\gamma_{2}-\gamma_{1}} e^{-\gamma_{1} u}+\frac{\gamma_{2}-\mu}{\gamma_{2}-\gamma_{1}} e^{-\gamma_{2} u} \\
\Psi(u) & =\frac{1-\gamma_{1} / \mu}{1-\gamma_{1} / \gamma_{2}} e^{-\gamma_{1} u}+\frac{1-\gamma_{2} / \mu}{1-\gamma_{2} / \gamma_{1}} e^{-\gamma_{2} u},
\end{aligned}
$$

The equality $\Psi(u)=\Psi_{c}(u)+\Psi_{c}(u)$ may be checked using $\gamma_{1}+\gamma_{2}=\tilde{c}+\mu, \gamma_{1} \gamma_{2}=$ $\tilde{c} \mu-\tilde{\lambda}$.

\section{Second-order Padé approximations for the Ruin PROBABILITIES OF THE PERTURBED AND CLASSIC Cramér-LundBerg PROCESSES}

To satisfy the boundary condition $\Psi(0)=\lim _{s \rightarrow \infty} s \hat{\Psi}(s)=1$, we must use at least a "two point" $(1,2)$ Padé approximation, since we must satisfy one condition as $s \rightarrow \infty$ as well. 
Alternatively, we may look for $(1,2)$ Padé approximations of the form

$$
\hat{\Psi}_{j}(s)=\frac{a_{j}}{s^{2}+b_{1} s+b_{0}}, \hat{\Psi}_{c}(s)=\frac{s+a_{d}}{s^{2}+b_{1} s+b_{0}}
$$

satisfying thus $\lim _{s \rightarrow \infty} s \hat{\Psi}_{j}(s)=\Psi_{j}(0)=0$, and $\lim _{s \rightarrow \infty} s \hat{\Psi}_{c}(s)=$ $\Psi_{c}(0)=1$. The Dufresne-Gerber equations (24) impose two more condition $b_{0}=\tilde{p} a_{d}, b_{1}=a_{d}+a_{j}+\tilde{p}$, leaving only two coefficients $a_{j}, a_{d}$ to determine.

We determine now the coefficients $a_{j}, a_{d}$ by equating the first coefficients in the Padé approximation of $\hat{\Psi}_{c}(s)$ around 0 , using $\hat{\bar{F}}(s)=$ $m_{1}-\frac{m_{2}}{2} s+\frac{m_{3}}{3 !} s^{2}+\ldots$

$$
\begin{aligned}
& \hat{\Psi}_{c}(s)=\frac{1}{s+\tilde{c}-\tilde{\lambda} \hat{\bar{F}}(s)}= \\
& \frac{1}{\tilde{p}+\left(1+\tilde{\lambda} \frac{m_{2}}{2}\right) s-\tilde{\lambda} \frac{m_{3}}{3 !} s^{2}+\ldots} \approx \frac{s+a_{d}}{s^{2}+\left(a_{d}+a_{j}+\tilde{p}\right) s+a_{d} \tilde{p}} \\
& \left(a_{d}+s\right)\left(\tilde{p}+\left(1+\tilde{\lambda} \frac{m_{2}}{2}\right) s-\tilde{\lambda} \frac{m_{3}}{3 !} s^{2}+\ldots\right) \approx s^{2}+\left(a_{d}+a_{j}+\tilde{p}\right) s+a_{d} \tilde{p}
\end{aligned}
$$

This yields:

$$
\left\{\begin{array}{l}
a_{j}=a_{d} \tilde{\lambda} \frac{m_{2}}{2} \\
a_{d}=\frac{3 m_{2}}{m_{3}}
\end{array}\right.
$$

Theorem 2. Second order approximations for the ruin probabilities of the Brownian perturbed Cramér-Lundberg process. a) Let

$$
a_{d}=\frac{3 m_{2}}{m_{3}}, a_{j}=a_{d} \tilde{\lambda} \frac{m_{2}}{2}=\frac{3 \lambda m_{2}^{2}}{\sigma^{2} m_{3}}
$$

and let $-\gamma_{1},-\gamma_{2}$ denote the roots of

$$
s^{2}+\left(a_{d}+a_{j}+\tilde{p}\right) s+a_{d} \tilde{p}=0
$$

Then:

(1) The discriminant of (26) is always non-negative. Assuming w.l.o.g. $\gamma_{1}<\gamma_{2}$, it holds that $0<\gamma_{1}<a_{d}<\gamma_{2}$.

(2) The approximations for the "creeping ruin" and "ruin by jump"

$$
\begin{aligned}
& \Psi_{c}(x)=\frac{a_{d}-\gamma_{1}}{\gamma_{2}-\gamma_{1}} e^{-\gamma_{1} x}+\frac{\gamma_{2}-a_{d}}{\gamma_{2}-\gamma_{1}} e^{-\gamma_{2} x} \\
& \Psi_{j}(x)=\frac{a_{j}}{\gamma_{2}-\gamma_{1}}\left[e^{-\gamma_{1} x}-e^{-\gamma_{2} x}\right],
\end{aligned}
$$


ON MATRIX EXPONENTIAL APPROXIMATIONS OF RUIN PROBABILITIESI5

satisfy (24), the first two conditions in $(23) \Psi_{c}(0)=1, \Psi_{j}(0)=$ 0 , fit the first two moments of the aggregate loss $L$, and are admissible.

(3) In terms of moments, the Laplace transforms are:

$$
\begin{aligned}
\hat{\Psi}_{c}(s) & =\frac{6 m_{2}+2 s m_{3}}{2 m_{3} s^{2}+\left(3 \lambda m_{2}^{2}+6 m_{2}+2 p m_{3}\right) s+6 p m_{2}} \\
\hat{\Psi}_{j}(s) & =\frac{3 \lambda m_{2}^{2}}{2 m_{3} s^{2}+\left(3 \lambda m_{2}^{2}+6 m_{2}+2 p m_{3}\right) s+6 p m_{2}}
\end{aligned}
$$

b) The input and stationary excess approximations are of the same form, with $a_{d}=m_{1}^{-1}, a_{j}=\lambda m_{1}$, and $a_{d}=\tilde{m}_{1}^{-1}=\frac{2 m_{1}}{m_{2}}, a_{j}=\lambda \tilde{m}_{1}=$ $\lambda \frac{m_{2}}{2 m_{1}}$, respectively.

Proof: a) Let us note that $s^{2}+\left(a_{d}+a_{j}+\tilde{p}\right) s+a_{d} \tilde{p}$ is negative at $s=-a_{d}$ (since $\left(a_{d}\right)^{2}+\left(a_{d}+a_{j}+\tilde{p}\right)\left(-a_{d}\right)+a_{d} \tilde{p}=-a_{d} a_{j}<0$, and $a_{d}$ and $a_{j}$ are positive. In particular, the discriminant must be non-negative and $\gamma_{1}<a_{d}<\gamma_{2}$.

Laplace inversion yields now (27). Furthermore, $\Psi_{j}$ is admissible by the assumption $\gamma_{1}<\gamma_{2}$ and $\Psi_{c}$ is admissible as sum of positive terms.

Remark 9. The geometric compound approximation in Theorem 2 may also be viewed as replacing the original process by a process with the same Brownian component and profit rate, with exponential claims of mean $m=\frac{m_{3}}{3 m_{2}}$, and modified claims intensity $\lambda^{\prime}=\frac{\lambda}{2} \frac{m_{2}}{m^{2}}$. This reduces when $\sigma=0$ to DeVylder's approximation for the classic CramérLundberg process!

Yet another DeVylder-type approximation may be obtained by equating the first four cumulants Seixas and dos Reis (2013). This yields the same profit rate, exponential claims of mean $m=\frac{m_{4}}{4 m_{3}}$, modified claims intensity $\lambda^{\prime}=\frac{\lambda}{6} \frac{m_{3}}{m^{3}}$ and volatility $\tilde{\sigma}^{2}=\sigma^{2}+\lambda\left(m_{2}-\frac{4}{3} \frac{m_{3}^{2}}{m_{4}}\right)$. Note this is not always admissible.

Remark 10. In the case of exponential claims of rate $\mu$, all three approximations are exact, reducing to the well-known formulas (Dufresne and Gerber, 1991). For example, with $\frac{\sigma^{2}}{2}=1, \lambda=\frac{1}{2}, p=1$, and exponential claims of rate 1 , the Cramér-Lundberg roots are $\gamma_{1}=1 / 2, \gamma_{2}=$ 
$2, a_{d}=1, a_{j}=\frac{1}{2}$, and the ruin probabilities are

$$
\begin{aligned}
\Psi_{j}(x) & =\frac{1}{3}\left(e^{-x / 2}-e^{-2 x}\right) \\
\Psi_{c}(x) & =\frac{1}{3} e^{-x / 2}+\frac{2}{3} e^{-2 x} \\
\Psi(x) & =\frac{2 e^{-x / 2}}{3}+\frac{e^{-2 x}}{3} .
\end{aligned}
$$

Theorem 3. Three second order approximations for the classic Cramér-Lundberg process. a) A Padé $(1,2)$ approximation of the claims transform:

$$
\begin{aligned}
\hat{f}(s) & =\frac{s\left(b_{1}-\mu_{1} b_{0}\right)+b_{0}}{s^{2} b_{2}+s b_{1}+b_{0}} \\
& =\frac{s\left(\mu_{3}-2 \mu_{1} \mu_{2}+\mu_{1}^{3}\right)+\left(\mu_{2}-\mu_{1}^{2}\right)}{s^{2}\left(\mu_{1} \mu_{3}-\mu_{2}^{2}\right)+s\left(\mu_{3}-\mu_{2} \mu_{1}\right)+\mu_{2}-\mu_{1}^{2}}
\end{aligned}
$$

gives rise to the following approximations for the equilibrium and ruin transform:

$$
\begin{aligned}
& \hat{f}_{e}(s)=\frac{1-\hat{f}(s)}{\mu_{1} s}=\frac{b_{0}+b_{2} / \mu_{1} s}{b_{0}+b_{1} s+b_{2} s^{2}} \Leftrightarrow \\
& \text { (29) } \hat{\Psi}(s)=\frac{\rho}{s} \frac{1-\hat{f}_{e}(s)}{1-\rho \hat{f}_{e}(s)} \approx \rho \frac{b_{2} s+b_{1}-b_{2} / \mu_{1}}{b_{2} s^{2}+b_{1}^{(D)} s+b_{0}^{(D)}}= \\
& \frac{\rho \bar{\mu}_{1}}{1-\rho} \frac{\left(\bar{\mu}_{3}-\bar{\mu}_{2}^{2}\right) x+\bar{\mu}_{2}\left(\bar{\mu}_{2}-1\right)}{\frac{\left(\bar{\mu}_{3}-\bar{\mu}_{2}^{2}\right)}{1-\rho} x^{2}+\left(\bar{\mu}_{3}+\frac{\rho \bar{\mu}_{2}^{2}-\bar{\mu}_{2}}{1-\rho}\right) x+\bar{\mu}_{2}-1}, x=s \mu_{1}
\end{aligned}
$$

where $b_{1}^{(D)}=b_{1}-\rho b_{2} / \mu_{1}, \quad b_{0}^{(D)}=(1-\rho) b_{0}$.

Let $\mu_{c}=3 \mu_{1} \mu_{2}-2 \mu_{1}^{3}+2 \sqrt{\left(\mu_{1}^{2}-\mu_{2}\right)^{3}}$. This approximation is weakly admissible iff

$$
\left\{\begin{array}{l}
\mu_{1}^{2}<\mu_{2}, \frac{\mu_{2}^{2}}{\mu_{1}}<\mu_{3}, \rho \in(0,1) \text { or } \\
\frac{\mu_{1}^{2}}{2} \leq \mu_{2} \leq \mu_{1}^{2},\left\{\begin{array}{l}
\mu_{3} \in\left(\mu_{c}, \frac{\mu_{2}^{2}}{\mu_{1}}\right) \text { or } \\
\mu_{3} \in\left(\frac{2 \mu_{2}^{2}}{3 \mu_{1}}, \mu_{c}\right), \rho \in\left(\frac{\mu_{1}\left(\mu_{c}+\mu_{3}\right)}{\mu_{2}^{2}-\mu_{1} \mu_{3}}, 1\right)
\end{array}\right.
\end{array}\right.
$$

b) Ramsay's stationary excess approximation, revisited. Imposing the correct limiting behavior $\lim _{s \rightarrow \infty} s \hat{\Psi}(s)=\Psi(0)=\rho=1 /(1+\theta)$, the Padé $(1,2)$ approximations for the equilibrium density and ruin 
transforms are:

$$
\begin{gathered}
\hat{f}_{e}(s) \approx \frac{N}{D}:=\frac{\tilde{b}_{0}+\left(\tilde{b}_{1}-\tilde{\mu}_{1} \tilde{b}_{0}\right) s}{\tilde{b}_{0}+\tilde{b}_{1} s+\tilde{b}_{2} s^{2}}, \\
\hat{\Psi}(s) \approx \rho \frac{D-N}{D-\rho N}:=\rho \frac{\tilde{b}_{2} s+\tilde{\mu}_{1} \tilde{b}_{0}}{\tilde{b}_{2} s^{2}+\tilde{b}_{1}^{(E)} s+\tilde{b}_{0}^{(E)}}, \\
\tilde{b}_{0}=\tilde{\mu}_{2}-\tilde{\mu}_{1}^{2}, \quad \tilde{b}_{1}=\tilde{\mu}_{3}-\tilde{\mu}_{2} \tilde{\mu}_{1}, \quad \tilde{b}_{2}=\tilde{\mu}_{1} \tilde{\mu}_{3}-\tilde{\mu}_{2}^{2}, \\
\tilde{b}_{1}^{(E)}=\tilde{b}_{1}(1-\rho)+\rho \tilde{\mu}_{1} \tilde{b}_{0}, \quad \tilde{b}_{0}^{(E)}=(1-\rho) \tilde{b}_{0},
\end{gathered}
$$

where $\tilde{\mu}_{i}$ are the factorially reduced moments of the equilibrium density.

c) The geometric compound approximation for $\psi(s)$, in terms of the stationary excess moments, is:

$$
\begin{aligned}
& \hat{\Psi}(s) \approx \frac{N}{D} \\
& N=s\left(-3 \tilde{m}_{2}^{3}+4 \tilde{m}_{1} \tilde{m}_{3} \tilde{m}_{2}-\tilde{m}_{1}^{2} \tilde{m}_{4}\right)-4 \tilde{m}_{3} \tilde{m}_{1}^{2}+6 \tilde{m}_{2}^{2} \tilde{m}_{1} \\
& D=s^{2}\left(-3 \tilde{m}_{2}^{3}+4 \tilde{m}_{1} \tilde{m}_{3} \tilde{m}_{2}-\frac{1}{2} \theta \tilde{m}_{4} \tilde{m}_{2}+\frac{2 \theta \tilde{m}_{3}^{2}}{3}-\tilde{m}_{1}^{2} \tilde{m}_{4}\right) \\
& +s\left(-4 \tilde{m}_{3} \tilde{m}_{1}^{2}+6 \tilde{m}_{2}^{2} \tilde{m}_{1}-\theta \tilde{m}_{4} \tilde{m}_{1}+2 \theta \tilde{m}_{2} \tilde{m}_{3}\right)+6 \theta \tilde{m}_{2}^{2}-4 \theta \tilde{m}_{1} \tilde{m}_{3} \\
& =s N+\frac{1}{6} \theta\left(36 \tilde{m}_{2}^{2}-3 s\left(s \tilde{m}_{4}-4 \tilde{m}_{3}\right) \tilde{m}_{2}+4 s^{2} \tilde{m}_{3}^{2}-6 \tilde{m}_{1}\left(4 \tilde{m}_{3}+s \tilde{m}_{4}\right)\right)
\end{aligned}
$$

or, in terms of the claims moments, is:

$$
\begin{aligned}
& N=2 \lambda\left(45 m_{4} m_{2}^{2}-60 m_{3}^{2} m_{2}+s\left(20 m_{3}^{3}-30 m_{2} m_{4} m_{3}+9 m_{2}^{2} m_{5}\right)\right) \\
& D=\left(40 \lambda m_{3}^{3}-60 \lambda m_{2} m_{4} m_{3}+12 p m_{5} m_{3}-15 p m_{4}^{2}+18 \lambda m_{2}^{2} m_{5}\right) s^{2} \\
& +\left(90 \lambda m_{4} m_{2}^{2}-120 \lambda m_{3}^{2} m_{2}+36 p m_{5} m_{2}-60 p m_{3} m_{4}\right) s-240 p m_{3}^{2}+180 p m_{2} m_{4} \\
& =s N+3 p\left(-80 m_{3}^{2}+4 s\left(s m_{5}-5 m_{4}\right) m_{3}-5 s^{2} m_{4}^{2}+12 m_{2}\left(5 m_{4}+s m_{5}\right)\right)
\end{aligned}
$$

Proof: a) After checking the formula $\hat{f}_{e}(s)=\frac{b_{0}+a_{1} s}{b_{0}+b_{1} s+b_{2} s^{2}}$, with $a_{1}=$ $b_{2} / \mu_{1}$, the formula (28) follows from the identity

$$
s \hat{\Psi}(s)=\rho \frac{1-\hat{f}_{e}(s)}{1-\rho \hat{f}_{e}(s)}=\rho \frac{b_{2} s^{2}+\left(b_{1}-a_{1}\right) s}{b_{2} s^{2}+\left(b_{1}-\rho a_{1}\right) s+b_{0}(1-\rho)}
$$

The weak-admissibility domain is provided in (Telek and Heindl, 2002).

Straightforward algebra yields b) and c) 


\section{WEAK ADMISSIBILITY AND COMPARISON OF THE APPROXIMATIONS FOR THE RUIN PROBABILITY OF THE CLASSIC CRAMÉR-LUNDBERG PROCESS WITH UNIFORM CLAIMS}

Definition 2. An approximation of the Pollaczek-Khinchine Laplace transform $\hat{\Psi}(s)$ will be called admissible if it implies a nonnegative input density $f(x)$ (and a non-increasing equilibrium density $f_{e}(x)$ ).

In actuarial science and queueing theory we only need finally the nonnegativity of the inverse of the Pollaczek-Khinchine Laplace transform, and this may hold even when the input density is not nonnegative.

Definition 3. a) An approximation of the Pollaczek-Khinchine Laplace transform $\hat{\Psi}(s)$ will be called weakly admissible if its inverse is nonnegative.

b) The weak admissibility domain I consists of all values of the compounding parameter $\rho$, and for Brownian perturbed processes, of all values of $\sigma$ that render an approximation weakly admissible.

Consider now Padé $(k-1, k)$ /exact $2 k-1$ moments fitting of the ruin probability of the classic Cramér-Lundberg process with uniform $U[0,1]$ claims. The Laplace transform of the claims is $\frac{1-e^{-s}}{s}$, and its moments are $m_{1}=\frac{1}{2}, m_{2}=\frac{1}{3}, m_{3}=\frac{1}{4}, \ldots, m_{i}=\frac{1}{i+1}$.

(1) In Mathematica for example, Padé $(1,2)$ fitting of the input transform $\frac{1-e^{-s}}{s}$ is achieved by aprlap=PadeApproximant $[(1-\operatorname{Exp}[-\mathrm{s}]) / \mathrm{s},\{\mathrm{s}, 0,\{1,2\}\}]$ $f=$ InverseLaplaceTransform[aprlap, s, t]

The first command yields: $\frac{1}{\frac{s^{2}}{12}+\frac{s}{2}+1}$, and the second yields:

$$
f_{1,2}(x)=4 \sqrt{3} e^{-3 x} \sin (x \sqrt{3}),
$$

which is inadmissible (becoming first negative at $x=\frac{\pi}{\sqrt{3}}$ ).

The input Pollaczek-Khinchine approximation derived from $\frac{1}{\frac{s^{2}}{12}+\frac{s}{2}+1}$ is:

$$
\hat{\Psi}(s)=\frac{(s+4) \rho}{s^{2}+(6-2 \rho) s+12(1-\rho)},
$$

and this is weakly admissible for all

$$
\rho \in(2 \sqrt{3}-3,1)=(0.464102,1)
$$

(the restriction comes from the inequality $b_{1} \geq 2 \sqrt{b_{2} b_{0}}$ ). 
ON MATRIX EXPONENTIAL APPROXIMATIONS OF RUIN PROBABILITIES19

(2) the Padé $(1,2)$ approximation of the stationary excess distribution is: $\frac{\frac{s}{15}+1}{\frac{s^{2}}{20}+\frac{2 s}{5}+1}=\frac{4(s+15)}{3\left(s^{2}+8 s+20\right)}$ (inadmissible), and the resulting stationary excess $(1,2)$ Pollaczek-Khinchine approximation

$$
\frac{(3 s+20) \rho}{3 s^{2}+4(6-\rho) s+60(1-\rho)}
$$

is weakly admissible for all

$$
\rho \in\left(\frac{3}{2}(-11+5 \sqrt{5}), 1\right)=(0.27051,1)
$$

(3) the geometric compound (1,2) Pollaczek-Khinchine approximation is:

$$
\frac{5(s+12) \rho}{(6-\rho) s^{2}+60 s+180(1-\rho)}
$$

and this has weakly admissible inverse for all

$$
\rho \in\left(\frac{1}{2}(7-3 \sqrt{5}), 1\right)=(0.145898,1)
$$

Remark 11. In this example, it is the geometric compound approximation which has the largest domain of weak admissibility.

On the other hand, none of the approximations is admissible. In fact, there exists no second order phase-type or matrix exponential density fitting the first two moments of the uniform, since the Aldous-Shepp (Aldous and Shepp, 1987) "Erlang bound" of $\frac{m_{2}}{m_{1}^{2}} \geq \frac{k+1}{k}$ does not hold for $k=2\left(\frac{4}{3}\right.$ is smaller than $\left.\frac{3}{2}\right)$

Similar comparisons could be undertaken for the Brownian perturbed process.

\section{REFERENCES}

Joseph Abate and Ward Whitt. An operational calculus for probability distributions via laplace transforms. Advances in Applied Probability, pages 75-113, 1996.

David Aldous and Larry. Shepp. The least variable phase type distribution is erlang. Stochastic Models, 3(3):467-473, 1987.

F. Andersson, M. Carlsson, and M.V. de Hoop. Sparse approximation of functions using sums of exponentials and aak theory. Journal of Approximation Theory, 163(2):213-248, 2011.

Soren Asmussen and Tomasz Rolski. Computational methods in risk theory: a matrix-algorithmic approach. Insurance: Mathematics and Economics, 10(4):259-274, 1992. 
Florin Avram and Miguel Usabel. Finite time ruin probabilities with one laplace inversion. Insurance: Mathematics and Economics, 32 (3):371-377, 2003.

A. Bobbio, A. Horváth, and M. Telek. Matching three moments with minimal acyclic phase type distributions. Stochastic models, 21(2-3): 303-326, 2005.

F. Dufresne and H.U. Gerber. Risk theory for the compound poisson process that is perturbed by diffusion. Insurance: mathematics and economics, 10(1):51-59, 1991.

Bogdan Dumitrescu, Bogdan C Sicleru, and Florin Avram. Semiinfinite optimization with sums of exponentials via polynomial approximation. arXiv preprint arXiv:1401.2305, 2014.

A.K. Erlang. The theory of probabilities and telephone conversations. Nyt Tidsskrift for Matematik B, 20:33-39, 1909.

Runhuan Feng. An operator-based approach to the analysis of ruinrelated quantities in jump diffusion risk models. Insurance: Mathematics and Economics, 48(2):304-313, 2011.

Esther Frostig, Susan M Pitts, and Konstadinos Politis. The time to ruin and the number of claims until ruin for phase-type claims. Insurance: Mathematics and Economics, 51(1):19-25, 2012.

J. Grandell. Simple approximations of ruin probabilities. Insurance: Mathematics and Economics, 26(2):157-173, 2000.

M.A. Johnson and M.R. Taaffe. Matching moments to phase distributions: Mixtures of erlang distributions of common order. Stochastic Models, 5(4):711-743, 1989.

V.V. Kalashnikov. Geometric sums: bounds for rare events with applications: risk analysis, reliability, queueing, volume 413. Springer, 1997.

Bara Kim and Jeongsim Kim. Higher moments of the waiting time distribution in $\mathrm{m} / \mathrm{g} / 1$ retrial queues. Operations Research Letters, 39(3):224-228, 2011.

Jeongsim Kim and Bara Kim. Waiting time distribution in an retrial queue. Performance Evaluation, 2013.

A.E. Kyprianou. Introductory lectures on fluctuations of Lévy processes with applications. Springer Verlag, 2006.

David Landriault, Tianxiang Shi, and Gordon E Willmot. Joint densities involving the time to ruin in the sparre andersen risk model under exponential assumptions. Insurance: Mathematics and Economics, 49(3):371-379, 2011.

Clyde Martin and Victor Shubov. Natural exponential families of probability distributions and exponential-polynomial approximation. Applied mathematics and computation, 59(2):275-297, 1993. 
ON MATRIX EXPONENTIAL APPROXIMATIONS OF RUIN PROBABILITIES21

M.F. Neuts. Matrix-geometric solutions in stochastic models: an algorithmic approach. Dover Pubns, 1981.

RD Nobel and HC Tijms. Waiting-time probabilities in the $\mathrm{m} / \mathrm{g} / 1$ retrial queue. Statistica Neerlandica, 60(1):73-78, 2006.

D. Potts and M. Tasche. Parameter estimation for nonincreasing exponential sums by prony-like methods. Preprint TU Chemnitz, 2012.

R. Prony. Essai éxperimental et analytique: sur les lois de la dilatabilité de fluides élastique et sur celles de la force expansive de la vapeur de l'alcool, a diférentes températures. Journal de l'École Polytechnique Floréal et Plairial, an III, 1(22):24-76, 1795.

P. Reinecke and $M$ Telek. Validity check of matrixexponentialdistributions. submitted, 2013.

Hanspeter Schmidli. Perturbed risk processes: a review. Department of Theoretical Statistics, University of Aarhus, 1999.

Miguel JM Seixas and Alfredo D Egidio dos Reis. Some simple and classical approximations to ruin probabilities applied to the perturbed model. In ACTUARIAL AND FINANCIAL MATHEMATICS CONFERENCE, page 69, 2013.

M. Telek and A. Heindl. Matching moments for acyclic discrete and continuous phase-type distributions of second order. Proc. of UK Performance Evaluation Workshop, UKPEW 2002, 2002.

Departement de Mathematiques, Universite de Pau, France

E-mail address: Florin.Avram@univ-Pau.fr

Department of Mathematics, Imperial College London, UK

E-mail address: m.pistorius@imperial.ac.uk 